\title{
Tracking Algorithm Based on Channel Propagating Characteristic in Wireless Sensor Network
}

\author{
Ying $\mathrm{Li}^{1,2}$, Yiliang $\mathrm{Wu}^{1}$, Nina $\mathrm{Hu}^{1}$, Guangsong Yang ${ }^{1, *}$ \\ ${ }^{1}$ School of Information Technology, Jimei University, Xiamen, China \\ ${ }^{2}$ Chengyi Colledge, Jimei University, Xiamen, China
}

\section{Email address:}

cyxxliying@jmu.edn.cn (Ying Li), 275948581@qq.com (YiliangWu), 1024166653@qq.com (Lina Hu), gsyang@jmu.edu.cn (Guangsong Yang)

${ }^{*}$ Corresponding author

\section{To cite this article:}

Ying Li, Yiliang Wu, Lina Hu, Guangsong Yang. Tracking Algorithm Based on Channel Propagating Characteristic in Wireless Sensor Network. International Journal of Intelligent Information Systems. Vol. 5, No. 5, 2016, pp. 65-70. doi: 10.11648/j.ijiis.20160505.12

Received: September 11, 2016; Accepted: October 11, 2016; Published: October 17, 2016

\begin{abstract}
In order to improve the mobile node tracking accuracy of indoor environment, a mobile node tracking algorithm based on channel propagating characteristic is proposed. Channel propagation model is established by actual measurement and fitting analysis in three different scenarios, which included closed corridor, open corridor and laboratory. The anchor node periodically measures the RSSI of the beacons from mobile node, to estimate the coordinates of the mobile node location, speed and direction by using the Maximum Likelihood method and channel model. The simulation results prove that the proposed scheme is effective and can meet the real-time requirements of indoor localization.
\end{abstract}

Keywords: Wireless Sensor Network, Propagating Characteristic of Channel, Tracking, Maximum Likelihood

\section{Introduction}

In some indoor scenarios, some specific targets should be tracked and positioned. For example, the specific seating in a meeting, the mobile support for people with visual disabilities, the pedestrian navigation system, the emergency monitoring automatic positioning alarm and the indoor robot control, etc. [1].

Reference [2] introduces a new sequence-detection method with maximum-likelihood-metrics for user movement tracking, which utilizes reference data and measured received signal strength indicator (RSSI) data to search for the most likely movement paths represented as sequences of indoor zones. Reference [3] formulates the sensor localization problem as a static parameter estimation problem for Hidden Markov Models and estimate these static parameters using a ML(Maximum Likelihood) approach. Reference [4] proposes a maximum likelihood reconstruction algorithm to reconstruct the node positions in a multi-dimensional Euclidean space based on only connectivity information. Both of these references did not consider the channel impact factors.
In real electromagnetic environment, especially indoor environment, there may exist some impact factors such as reflection, scattering and multi-path due to the different building structure and building materials [5]. Therefore, it is not suitable to use positioning methods in times and angles. For RSSI localization algorithm, the strength of the received waves not only depends on the distance between nodes, but also on the affects of the channel propagation characteristics.

Classical propagation models include Rayleigh fading model and Rice distribution model etc. They both have some flaws in indoor environment. Rayleigh fading model describes the decline of small amplitude fluctuation in rapid changing. It assumes that the strengths of all the signals reaching the receiver are the same, regardless of the direct electromagnetic wave. There are differences between this method and the reality. In addition to the analysis of the path of the low level dispersion, Rice distribution model mainly considers the existence of a stable direct path. The model needs to be physically isolated from the direct waves. 
Therefore, it's somewhat difficult to determine the propagation parameters.

Therefore, in order to improve the positioning accuracy, it is necessary to research on the characteristics of wireless transmission channel and work out the statistics of the probability distribution of wave intensity. Monitor the location of the object on the basis of the probability model. Reference [6] proposed a positioning method combine with the sensor network node and RFID by considering the WAF experience model [7].The positioning method for long and narrow space based on the Human Body Penetration Loss Model is proposed in [8], which skill fully combined RSSI ranging with extended Kalman filter positioning algorithm.

The indoor localization algorithm LANDMARC based on RSSI is proposed in [8], which can improve the positioning accuracy by combine the signal strength indication of the virtual reference tags determined by the distance-loss model [9].

In our paper, to study the Channel propagation model in indoor environment, we do different experiments in several scenarios, to set up channel propagation model by channel statistics probability, improve the positioning accuracy by Maximum Likelihood method.

The organization of the paper is as follows. We begin with theIntroduction of the Maximum Likelihood and the channel propagation modelingin Section 2. In Section 3 we proposed a tracking and position algorithm based on channel propagating characteristic. In Section 4 we give the result of our experiment in three different scenarios. In section 5 we do some simulation experiments to evaluate the performance of our proposed method. In Sections 6 we provide a discussionand a few concluding remarks.

\section{The Maximum Likelihood Method and the Propagation Modeling}

\subsection{Maximum Likelihood}

When we infer the value of $\theta$ from $x_{1}, x_{2}, \ldots x_{n}$, if $x_{1}, x_{2}, \ldots x_{n}$ most likely to appear, $\theta$ is the best. Therefore, the likelihood function can be defined as:

$$
L(\theta)=L\left(\theta ; x_{1}, \ldots x_{n}\right)=f\left(x_{1}, \ldots, x_{n} ; \theta\right)
$$

If we consider $\theta$ as a variable of function $f\left(x_{1}, x_{2}, \cdots x_{n} ; \theta\right)$, we can obtain the value of $\theta$ which can maximizes the likelihood function.

If $x_{1}, x_{2}, \ldots x_{n}$ is independent, it satisfies the following condition

$$
\begin{gathered}
f\left(x_{1}, \cdots, x_{n} ; \theta\right)=\prod_{i=1}^{n} f\left(x_{i} ; \theta\right) \\
L(\theta)=L\left(\theta ; x_{1}, \cdots, x_{n}\right)=\prod_{i=1}^{n} f\left(x_{i} ; \theta\right)
\end{gathered}
$$

Therefore, we can obtain the value of $\theta$ through the equation (4).

$$
\frac{\partial}{\partial \theta} \log L(\theta)=0
$$

Of course, it also can transform the $L(\theta)$ totheform of $\log L(\theta)$. When there exist $k$ parameters $\theta=\left(\theta_{1}, \theta_{2}, \cdots, \theta_{K}\right)$, we can solve equation (5) to obtain $\theta$.

$$
\frac{\partial}{\partial \theta_{i}} \log L(\theta)=0 \quad(1 \leq i \leq k)
$$

\subsection{The Propagation Modeling}

In the actual environment, the characteristics of channel are changingdynamic, so it is necessary to do some experiments to measure the channel with probability statistics. Firstly, we change the distance between the communication nodes, and measure received signal strength to get the relationship between the distance and signal strength. Secondly, we model the channel propagation characteristics according to the results of our experiments. Assume the distance between the send and receive wireless node is $d, \theta$ can be obtained when $L(\theta)$ is maximized.

In our model, the average received power strength $P a$ can be expressed in (6),

$$
P a=C d^{-a}
$$

Where $C$ is a constant and $\alpha$ is an attenuation coefficient. Sampling and measurement of multiple $(d, P)$ groups, $C$ and $\alpha$ can be obtained.

To describe the channel characteristics more accurately, assume $z=P / P a$ obeys Weibull distribution as shown in equation (7),

$$
f(z)=\beta m z^{m-1} e^{-\beta z^{m}} \quad(z \geq 0 ; m, \beta>0)
$$

Fromn samples $\left(d_{1}, P a_{1}\right),\left(d_{2}, P a_{2}\right), \ldots,\left(d_{n}, P a_{n}\right) \quad$ and equation (7), we can get $z_{1}, z_{2}, \ldots z_{n}$. With parameter $\theta=(\beta, m)$ and the likelihood function $L(\theta)=f\left(z_{1}, z_{2}, \ldots z_{n} \mid \theta\right)=f\left(z_{1} \mid \theta\right) \ldots f\left(z_{n} \mid \theta\right)$, to determine the parameters of $m$ and $\beta$ in (7) by likelihood calculation.

\section{Tracking and Position Algorithm Based on Channel Propagating Characteristic}

\subsection{Network Model}

$\mathrm{T}$ Network model is shown in Figure 1. Assuming that there are $\mathrm{n}$ fixed anchor nodes in study area, they can communicated between each other by wired or wireless methods, finally the collected data is converged to the Sink for centralized processing. 


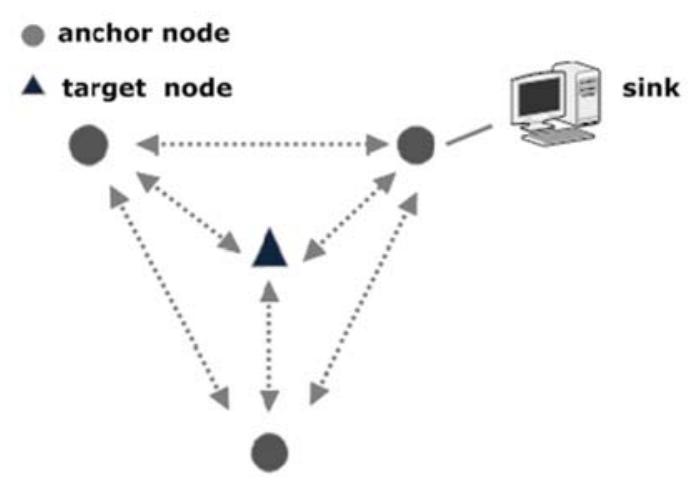

Figure 1. Network model.

Each moving target node has only network IDand send beacons to anchor nodes with the same power periodically. After receiving the information from target nodes, anchor node transmits them to the Sink node.Sink node can speculate the channel condition and target node information according to the accurate position of each anchor node and the received information from target node $T_{j}(j=1,2,3, \ldots, m)$, such as ID, receiving time, receiving power of the mobile node to the target. Sink speculates the location based on the received information and specific algorithm. Considering the power consumption of moving targets, generally, the complex algorithm of the position speculation is carried out at anchor nodes.

\subsection{The Location and Tracking Method Based on the Maximum Likelihood Method}

In this section, we proposed a location and tracking method based on the maximum likelihood method, which comprehensively takes channel characteristic, target node position, speed and direction into consideration.

Mobile terminal is also called target node, it keeps moving whilesend $n$ beacons within a specified time interval periodically which are to be received by $j$ anchor points. At this point, the ith beacon sent by the target is received by $j \quad\left(x_{a, j}, y_{a, j}\right)$ anchor, and the received power is $P_{i j}(1 \leq i \leq l, 1 \leq j \leq n)$.

Assume a target is doing uniform motion in a straight line with the speed of $v$ and thedirection angle of $a$. When speculate position, the actual location of the target is denoted as $(x, y)$. The difference between the moment of the $i$ beacon is sent and speculate time is denoted as $\Delta t_{i}$.The $j$ anchor points received power from beacon $i$ is $P_{i j}$. The conditional probability density is $p\left(P_{i, j} \mid v, a, x, y\right)$ which can be obtained according to equation (5) and (6),

$$
\begin{gathered}
x_{i}=x-v \Delta t_{i} \cos (a) \\
y_{i}=y-v \Delta t_{i} \sin (a) \\
d_{i j}=\sqrt{\left(x_{i}-x a_{j}\right)^{2}+\left(y_{i}-y a_{j}\right)^{2}} \\
P_{i j}=C d_{i j}^{-\alpha}
\end{gathered}
$$

$$
\begin{aligned}
z_{i j} & =P_{i j} / P a_{i j} \\
p\left(P_{i j} \mid v, a, x, y\right) & =f\left(z_{i j}\right)=\beta m z_{i j}{ }^{m-1} e^{-\beta z i, j}{ }^{m}
\end{aligned}
$$

The power of ith beaconreceived by $j$ anchor node is a

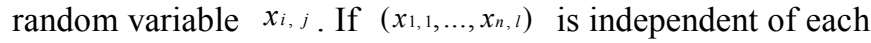
other, take $\theta=(v, a, x, y)$, we can solve the target position, speed, and directionwith the likelihood function of equation (14) and maximum likelihood method.

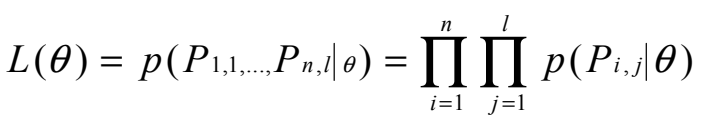

\section{The Measurement of the Channel Models}

According to the measurement method in reference [10], we do our experiments in the following three scenarios show as Figure 2, Figure 3 and Figure 4.

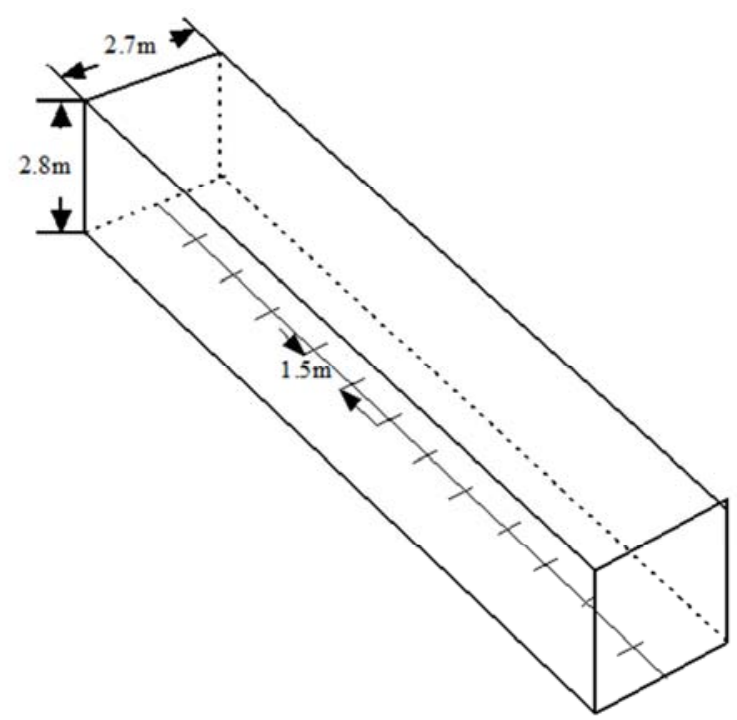

Figure 2. Closed corridor.

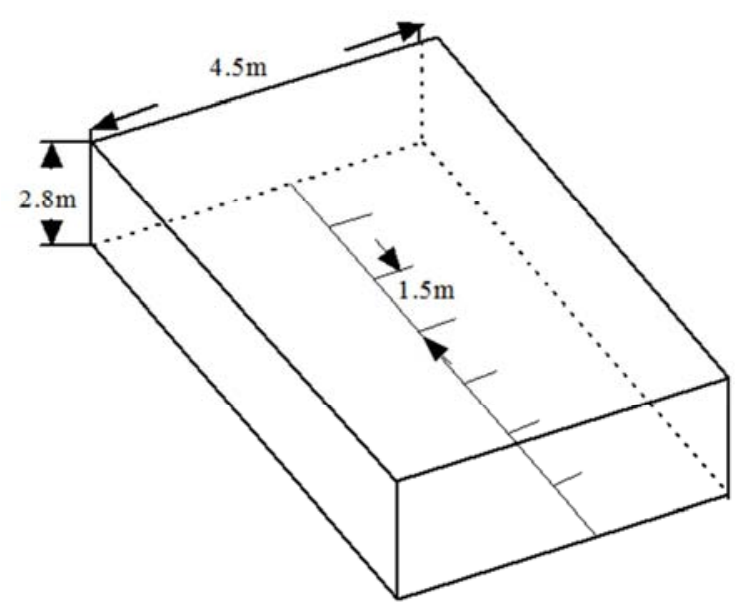

Figure 3. Open corridor. 


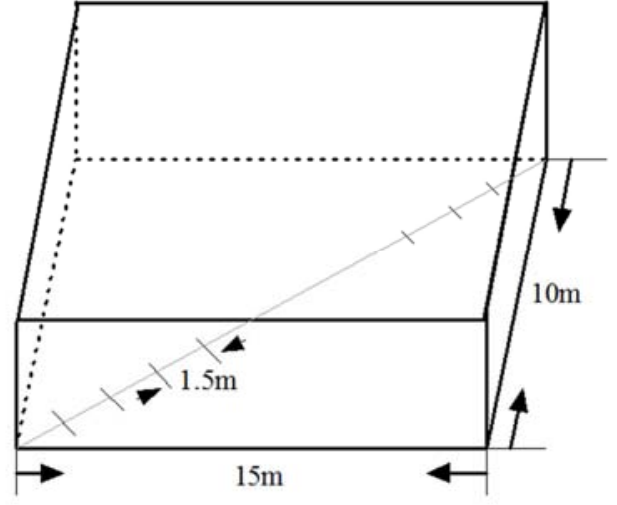

Figure 4. Closed corridor.

Scenario 1, a closed corridor with walls on both sides which high 2.8 meters and wide 2.7 meters. In the middle of the corridor, conduct one signal measurement every 1.5 meters.

Scenario 2, a opening corridor, on one side of the open, on the other side there is a wall with 2.8 meters height and 4.5 meters width. Conduct one signal measurement every 1.5 meters along in the middle of the corridor.

Scenario 3, a laboratory, equipped with furniture and computer, with 2.8 meters height and 15 meters length and 10 meters width. Conduct one signal measurement every 1.5 meters along the laboratory diagonal.

The transmitter is a wireless router D-Link with the IEEE $802.11 \mathrm{~b}$ protocol, the frequency is set $2.4 \mathrm{GHz}$, and rate is 11 Mbps. The receiver is a laptops with Wireless Mon software [11] [12] to measure the signal strength. In each scenario, the receiving strength in different distance between sending and receiving nodes is measured in $2.5 \mathrm{~m}$ transmit-receive separation, the test results are the average of 10 groups, and show in Figure5.

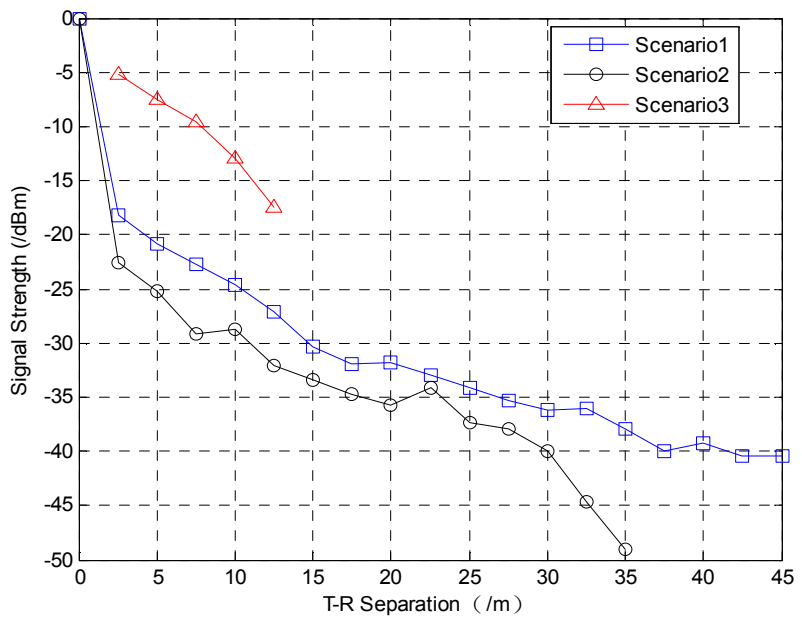

Figure 5. The relationship between the signal strength and transmit-receive separation.

According to (6) and (7) to calculate the transmission characteristics, the results are shown in table 1. The probability distribution of three scenarios propagation characteristics are shown as Figure 6, Figure 7, and Figure 8.
Table 1. Channel propagation characteristics under three different scenarios

\begin{tabular}{llll}
\hline & $\begin{array}{l}\text { Scenario 1 } \\
\text { (closed corridor) }\end{array}$ & $\begin{array}{l}\text { Scenario 2 } \\
\text { (open corridor) }\end{array}$ & $\begin{array}{l}\text { Scenario 3 } \\
\text { (laboratory) }\end{array}$ \\
\hline $\mathrm{C}$ & $9.647 \mathrm{e}-05$ & $2.238 \mathrm{e}-05$ & 0.0007621 \\
$\mathrm{a}$ & 1.666 & 1.463 & 0.9876 \\
$\mathrm{~m}$ & 2.501 & 2.262 & 2.98 \\
$\mathrm{Beta}$ & 0.004197 & 0.006092 & 0.003801 \\
\hline
\end{tabular}

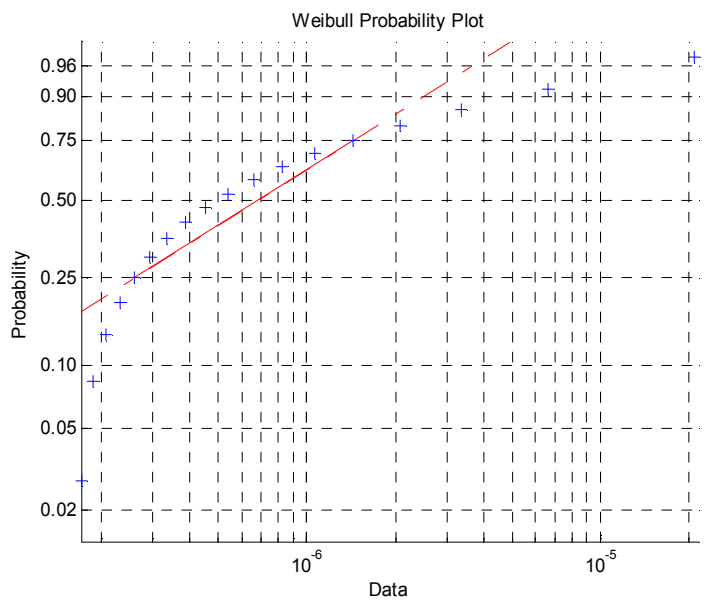

Figure 6. Weibull probability distribution in the closed corridor.

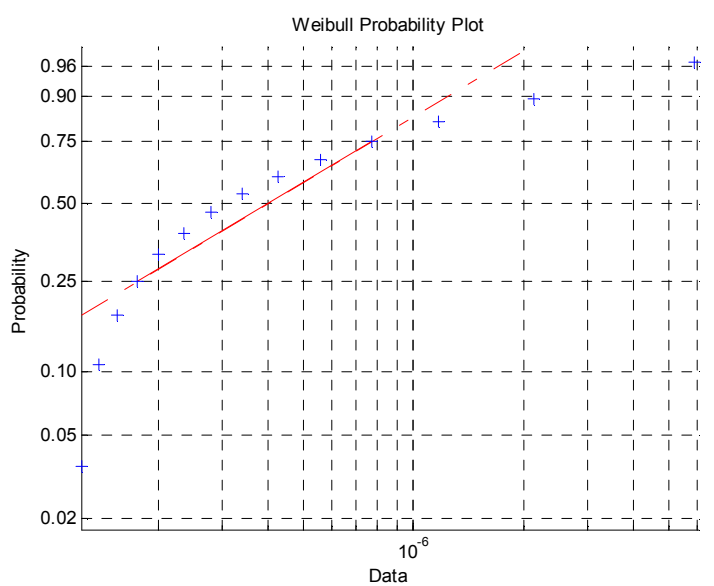

Figure 7. Weibull probability distribution in the open corridor.

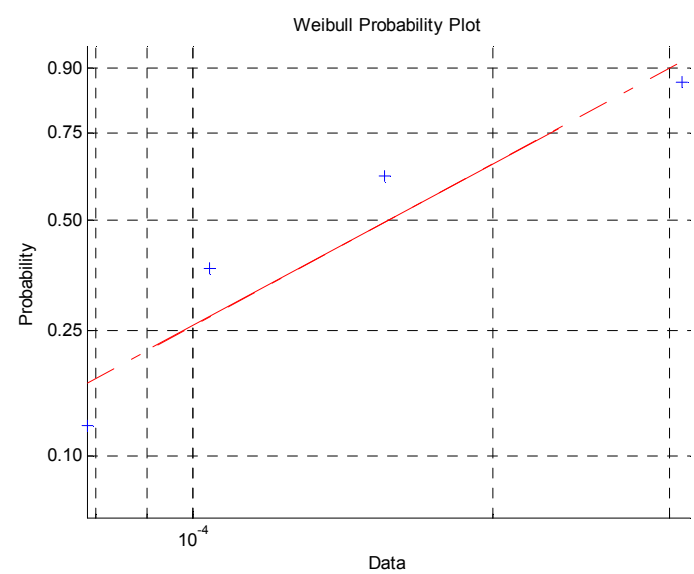

Figure 8. Weibull probability distribution in the laboratory. 


\section{Algorithm Simulation and Evaluation}

In this section, we use the measure result in section 3 to evaluate the performance of our proposed tracking algorithm in section 2 by simulation experiment in different scenarios.

For scenario 1 (Figure 2) and scenario 2 (Figure 3), the target nodes move from the one of the endpoints to the other endpoint of the corridor respectively. Anchor node is set respectively in two endpoints and the middle point. In scenario 3 (Figure4), the target node moves along the diagonal, the anchor nodes are set in the four corners of the laboratory, and the movement speed is $1 \mathrm{~m} / \mathrm{s}$. Using the proposed algorithm in 2.2, collect data per 1.5 seconds, and speculate the trajectory of the mobile node.

Take a period of time to carry out statistics randomly, Figure 9 to Figure 11 are the estimations of speed, angle and node coordinate error during the periods from 12 to 18 seconds. It can be seen from Figure 9 that the movement speed of target nodes are all close to the actual speed $1 \mathrm{~m} / \mathrm{s}$, in open corridor scenarios is higher than that in the closed corridor and laboratory scenario. With the increase of mobile time, move speed increased slightly after 13.5 seconds, and gradually stabilized after 16.5 seconds. Among them, movement speed in scenario 3 deviates the most from reality, while the movement speed in scenario 2 is closest to the real one.

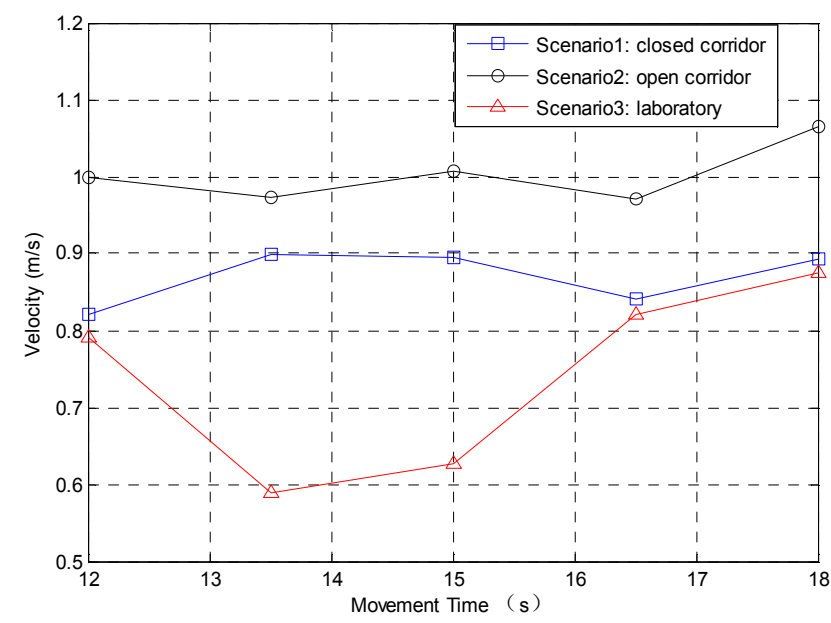

Figure 9. The comparison of the mobile node speed in three different scenarios.

Figure 10 shows the angle difference of three scenes leveled off and became close to realistic Angle. Figure11 shows that with the increase of mobile time, the errors between the mobile node coordinates and the actual coordinates measurement of the three scenes are as follows: for "scenario 1" the error is between $1.4 \mathrm{~m}$ to $2.8 \mathrm{~m}$. For Scenario 2the error is between $0.1 \mathrm{~m}$ to $1.2 \mathrm{~m}$. For Scenario 3 the error is between $0.8 \mathrm{~m}$ to $1.9 \mathrm{~m}$. Scenario 1 is greater than Scenario 3 which is greater than Scenario 2 considering the error between measured coordinate with actual coordinate.

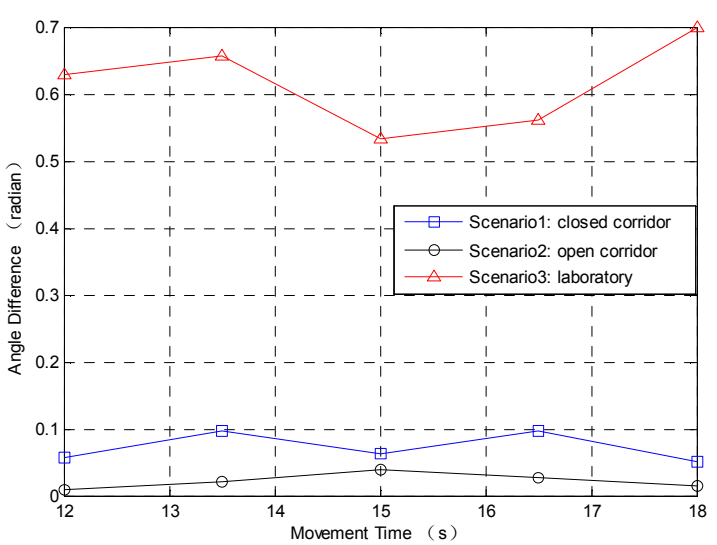

Figure 10. The comparison of the mobile node angle difference in three different scenarios.

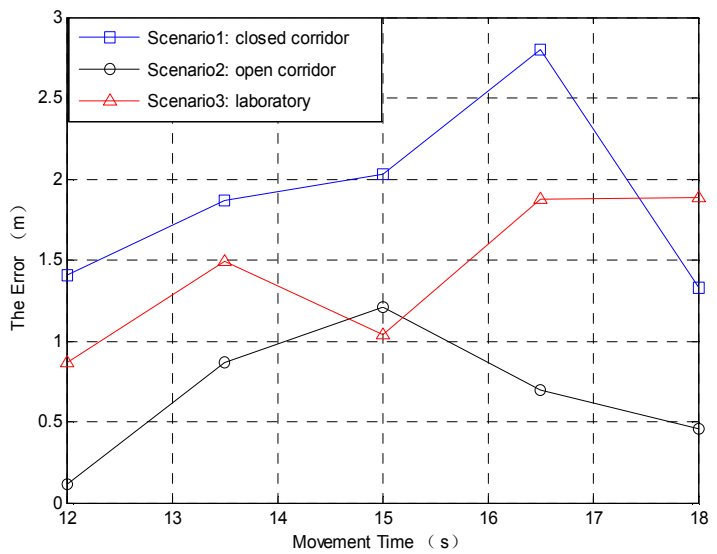

Figure 11. The comparison of the mobile node error in three different scenarios.

\section{Conclusion}

The paper mainly introduced the mobile node maximum likelihood tracking algorithm and applied it into practice in three different scenarios, an closed corridor, an open corridor and a laboratory, calculated its propagation characteristics, and analyzed the differences and conduct relevant performance evaluation through the MATLAB software.

The proposed scheme estimates the coordinates of the mobile node location through regularly collecting sampling measurement of mobile node beacon, strength of anchor nodes received by mobile node to send signals and anchor node itself. The likelihood function was established based on the sampling values. The problem is transfer into figure out the target speed $v$, direction $a$, and the target position $(x, y)$ when the likelihood function is max.Using maximum likelihood method of mobile node position tracking algorithm for mobile nodes and Matlab software simulation proves it is feasible under three different scenarios: the closed corridor, the open corridor and the laboratory. The calculation result is in accordance to the actual value.

In our future work, we will use the real hardware sensor nodes, such as ZigBee technology, to do some experiments, to further verify the proposed algorithm. 


\section{Acknowledgements}

This work is supported by Science Foundation of Fujian Province (No.2015J01267, No.2015J01657), Science Foundation of Fujian Education Department (No.JA15650, No.JA15257), Science Foundation of Xiamen City (No.2014S0884, No. 3502Z20153010).

\section{References}

[1] NasrullahPirzada, M YunusNayan,FazliSubhan, etal. Device-free localization technique for indoor detection and tracking of human body: A survey. International Conference on Innovation, Management and Technology Research (ICIMTR). Malaysia: Procedia-Social and Behavioral Sciences,2014,129:pp.422-429.

[2] PiotrWawrzyniak,SławomirHausman,PiotrKorbel.Area based indoor tracking algorithm based on sequence detection and maximum likelihood metrics.2016 10th European Conference on Antennas and Propagation, 2016,pp.1-3.

[3] Nikolas Kantas,Sumeetpal S. Singh,Arnaud Doucet.Distributed Maximum Likelihood for Simultaneous Self-Localization and Tracking in Sensor Networks.IEEE Transactions on Signal Processing.2012,pp.5308-5047.

[4] Sonia A. Bhaskar.Localization From Connectivity: A 1-bit Maximum Likelihood Approach.IEEE/ACM Transactions on Networking.2015,pp.1-15.
[5] K.J.Mao, J.B.Wu. Indoor Localization Algorithm for NLOS Environment. Acta Electronica Sinica, 2016,pp.1174-1179.

[6] G.A. Gonçalo. Novel Approach to Indoor Location Systems Using Propagation Models in WSNS. International Journal on Advances in Networks and Services, 2015, 4(2),pp.251-256.

[7] P. Bahl,V. N. Padmanabhan.RADAR: An In-building RF-Based user Location and Tracking System.in Proc. IEEE INFOCOM, 2000,pp.775-784.

[8] Y.Z.WANG. Research and Application of RFID Location Algorithm based on Reference tags. Journal on Communications, 2010, 31(2),pp. 86-92.

[9] J. Ying. Boundary Virtual Reference Tags Location Algorithm Based on RFID. Computer Engineering,2011,37(6), pp.274-276.

[10] A. Robert, D. Tummala,Xinrong Li. Indoor Propagation Modeling at2.4 GHZ for IEEE 802.11.The Sixth lasted International Multi-Conference on Wireless and Optical Communications Wireless Networks and Emerging Technologies.July3-5 2006.

[11] PASSMARK SOFT WARE.Wireless Mon.2016,03.10.http://www.wirelessmon.com.

[12] M.Milner, Network Stumbler Version 0.40.2001-2004,Netstumbler, http://www.netstumbler.com. 